\title{
Fluoroscopy-Guided Transforaminal versus Caudal Epidural Steroid Injection for Chronic Pain After Spinal Surgery: A Retrospective Mid-Term Comparative Study
}

\author{
Jun Hyeong Song' \\ Woo Yong Lee ${ }^{2}$ \\ Kyoung Rai Cho ${ }^{3}$ \\ Sang Hyun Nam (iD ${ }^{4}$ \\ Ki Deok Park ${ }^{5, *}$ \\ Yongbum Park (iD ${ }^{1, *}$ \\ 'Department of Physical Medicine \& \\ Rehabilitation, Sanggye Paik Hospital, Inje \\ UniversityCollege of Medicine, Seoul, \\ Republic of Korea; ${ }^{2}$ Department of \\ Anesthesiology, Sanggye Paik Hospital, \\ Inje University College of Medicine, \\ Seoul, South Korea; ${ }^{3}$ Department of \\ Otorhinolaryngology Head and Neck \\ Surgery, Sanggye Paik Hospital, Inje \\ University College of Medicine, Seoul, \\ Republic of Korea; ${ }^{4}$ Department of \\ Plastic and Reconstructive Surgery, \\ Sanggye Paik Hospital, Inje University \\ College of Medicine, Seoul, Republic of \\ Korea; ${ }^{5}$ Department of Rehabilitation \\ Medicine, Gachon University, Gil Medical \\ Center, Incheon, South Korea
}

*These authors contributed equally to this work

Correspondence: Yongbum Park Department of Physical Medicine \& Rehabilitation, Sanggye Paik Hospital, Inje University College of Medicine, Sanggye 7 Dong 76I-7, Nowon-gu, Seoul, 139-707, Korea

Email swc328@naver.com

Ki Deok Park

Department of Rehabilitation Medicine, Gachon University, Gil Medical Center,

Namdong-Daero 774 Beon-gil,

Namdong-gu, Incheon, Korea, 33

$\mathrm{Tel}+82-2-950-$ I I 45

Fax +82-2-935-3076

Email bduck@gilhospital.com
Introduction: This study is to compare advantages, safety and mid-term effects of fluoroscopy (FL)-guided transforaminal (TF) to caudal (CA) epidural steroid injection (ESI) for chronic pain after spinal surgery (CPSS) by assessing pain relief and improvement of functionality.

Methods: Patients with radicular pain in CPSS who received FL-guided CA $(n=21)$ or TF $(n=28)$ ESI were included in this retrospective study. Complication frequencies, adverse events, treatment effects, and functional improvements for each procedure were compared at 1,3 , and 6 months following the last injection.

Results: Both the Oswestry Disability Index (ODI) and verbal numeric pain scale scores (VNS) demonstrated improvement in both groups at all 1, 3, and 6 months following the last injection, without meaningful difference between groups $(\mathrm{p}<0.05)$. Moreover, no meaningful difference was present between groups in terms of treatment success rate at every time point. The amount of time used for the injection procedure was shorter in CA group than in TF group $(410.32 \pm 25.73$ seconds vs $640.65 \pm 18.03$ seconds, $\mathrm{p}<0.05)$. Within 2 weeks of the injection treatment, the patient satisfactory scores were evaluated, with excellent being rated $85.7 \%(\mathrm{n}=18)$ among CA-ESI patients and 55.7\% $(\mathrm{n}=16)$ among TF-ESI patients $(\mathrm{p}$ $<0.05$ ). Logistic regression analysis revealed that variables such as method of injection (CA or TF approach), sex, use of analgesics, pain duration, number of injections, and age were not significant variables for successful treatment results. There were no adverse complications after the procedure in both groups.

Conclusion: The outcomes of FL-guided CA-ESI and TF-ESI for CPSS are similar in terms of pain reduction and functional improvements. CA-ESI is associated with lesser procedure time. In addition, compared with the TF-ESI, the patient experiences less discomfort during the injection, and the satisfaction with the injection treatment is confirmed to be better. Accordingly, both methods are effective; however, in the CA approach, patient satisfaction is higher and the procedure time is shorter.

Keywords: epidural block, fluoroscopy, transforaminal, caudal, FL, CA, TF

\section{Introduction}

Failed back surgery syndrome was recently renamed, as chronic pain after spinal surgery (CPSS) by international classification of disease-11. ${ }^{1}$ CPSS is chronic back pain or leg pain developing after surgery that persists or recurs for longer than 3 months following spinal surgery (i.e., beyond the healing process). The pain is either localized to the back or projected into one or both limbs. ${ }^{1,2}$ 
CPSS is commonly described after spinal stenosis or disc herniation surgery. ${ }^{1-4}$ About $20 \%$ of patients undergoing lumbar spinal surgery develop pain which requires additional surgery or other interventions to alleviate pain. ${ }^{1-5}$ CPSS patients are significantly disabled in function and report poor quality of life, worse than other chronic pain conditions. ${ }^{1-3}$ CPSS has been hypothesized to be secondary to multiple factors such as epidural fibrosis, acquired stenosis, sacroiliac joint pain, and facet joint pain. ${ }^{6}$ CPSS, which is non-responsive to conservative management with rehabilitation techniques and medication, is managed by epidural steroid injections (ESIs). ${ }^{6-9}$ ESIs reduce pain induced by epidural fibrosis, recurrent disc herniation and other etiologies. In previous studies, Caudal (CA)-ESI showed effectiveness with over $55 \%$ of improvement in functional status and significant pain relief in $60 \%$ to $70 \%$ in chronic back pain patients, including CPSS patients. ${ }^{8}$ In addition to CA-ESI, transforaminal (TF)-ESI may be effective in patients with CPSS who have radicular pain. Fluoroscopy (FL)-guided TF-ESI is considered to be precise and effective route of administration. ${ }^{10}$ The TFESI is advantageous because corticosteroid is injected closer to irritated nerve root which is a probable pain source. Also, TF-ESI results in better ventral epidural spreading than CA-ESI. ${ }^{10}$ However, few studies have compared the two injection methods in CPSS patients. Hence, the aim of this study is to evaluate mid-term treatment effect and safety of 100 FL-guided TF-ESI and CA-ESI in the case of CPSS with unilateral lower lumbar radicular pain through a retrospective comparative study. In addition, the factors that may affect the treatment effect were examined.

\section{Materials and Methods Study Design}

The present study is a case-controlled, retrospective study of chart data in electronic medical recording. Approval from the Institutional Review Board of Sanggye Paik Hospital was obtained. Informed consent was waived because there was no direct contact with the study population during the study period. Patient privacy and data confidentiality were maintained throughout the research. All patient identifiers were removed from the data set on initial collection. This study was conducted in accordance with the Declaration of Helsinki.

\section{Subject}

Patients who underwent FL-guided TF or CA ESI with diagnosis of CPSS from January 2015 to December 2018 were recruited through electric chart review. Ninety-four patients with unilateral radicular pain at lower extremity and back pain due to CPSS were referred to our pain clinic during the study period. Diagnosis of CPSS are based on Diagnostic Criteria of CPSS in ICD-11. ${ }^{1}$ Those who met the following inclusion criteria were selected: aged 18 or older and patients with radiating pain refractory to anti-inflammatory medications, analgesic or physical therapy for at least 3 months. Also, absence of progressive motor deficit or significant sensory deficit, cauda equina syndrome, revision operation or other invasive procedures had to be confirmed before inclusion. The exclusion criteria included a diagnosis of sacroiliac joint or facet joint pain, psychiatric disorders, and systemic inflammatory disease such as rheumatoid disorders.

\section{Fluoroscopic Intervention}

FL-guided TF or CA-ESI for CPSS patients with unilateral lower lumbar radicular pain was a common practice in our service. After receiving information about the overall procedure, potential risks, benefits of the intervention and risks of corticosteroid mixed with contrast media patients were asked to fill out consent form. Two physicians (Y. Park, YW. Lee) with more than 10 years of experience in FL-guided procedures performed the entire intervention. All procedures were performed on an outpatient basis.

We performed ultrasonography before injection of the CA-ESI in all patients. The reason is that if the diameter of sacral canal is less than $2 \mathrm{~mm}$, a needle larger than 22 gauge needle cannot be used. In addition, because there are cases of closed sacral hiatus in some patients, CA-ESI cannot be performed in this case.

For FL-guided CA-ESI, the patients lay prone on a fluoroscopic table. A pillow was placed under their hips to tilt the pelvis, bringing the sacral hiatus into greater prominence. After sanitizing the sacrococcygeal area with iodine-based povidone solution and alcohol solution, the physician localized the tip of the coccyx through palpation with sterile gloves. The anteroposterior view was adjusted for the optimal view of sacral hiatus. A 22-gauge, 3.5-inch length spinal needle $\left(\operatorname{Spinocan}^{\circledR}\right.$, BRAUN, Melsungen, Germany) was inserted into the epidural space using the image intensifier. The syringe was observed for blood before further advancement and inhalation was performed to check for blood or cerebrospinal fluid regurgitation. To confirm 
needle placement in the epidural space and avoid intravascular, intradural, or soft tissue infiltration, approximately $1 \mathrm{~mL}$ of contrast media (Omnipaque 300; GE Healthcare, Carrigtwohill, Co. Cork, Ireland) was injected before drug injection (Figure 1A). After a detailed review of epidurogram, 1-2 mL of 1\% lidocaine was injected as a test dose, followed by monitoring for the onset of clinical manifestations indicating intravenous analgesic influx such as metallic taste, dizziness, tachycardia, auditory changes, slurred speech, and motor ataxia for 1 to 2 minutes. Then, the drug consisting of $20 \mathrm{cc}$ mixture of $0.5 \%$ lidocaine $18.0 \mathrm{~mL}$ and dexamethasone $10 \mathrm{mg} 2 \mathrm{~mL}$ was injected.

For FL-guided TF-ESI, all patients were positioned to lie prone with a pillow between the lower abdomen and iliac crest to reduce lumbar lordosis. The $\mathrm{C}$-arm was adjusted so that the $\mathrm{x}$-ray is transmitted vertically to the endplate of the lower portion of the upper vertebral body. After confirming the trajectory view from the oblique view of the C-arm, the point directly inferior to the pedicle was set as the target. Aseptic dressing at the presumed needle insertion site with betadine and alcohol was done. The tip of a 23-gauge, 3.5-inch spinal needle was gradually advanced from the 6 o'clock direction, toward the target inferior to the pedicle under radiologic guidance. During the procedure, we ensured that the tip was placed in the inferolateral area of the intraarticular space confirmed by several radiologic views. When the needle tip touched the inferolateral border, the lateral view was obtained, and the needle was advanced in anterior and superior direction of the neural foramen. Optimal needle placement for injection into the anterior epidural space was confirmed by injecting small volume of radio contrast media under intermittent fluoroscopic imaging (Figure 1B). The test dose of $1 \%$ lidocaine was injected, and just like CA- ESI, the onset of side effects caused by intravenous analgesics was monitored. If none were observed $3 \mathrm{cc}$ mixture consisting of $0.5 \%$ lidocaine $1 \mathrm{~mL}$ and dexamethasone $10 \mathrm{mg} 2 \mathrm{~mL}$ was injected. In the case of TF-ESI with multiple levels of suspected lesion, selective nerve root block was performed before epidural block in consideration of CT, MRI, and clinical symptoms to determine the injection site. If the back and radicular pain improved more than $50 \%$ after the injection, it was diagnosed as the causative site and subsequently treated with TF-ESI. In both groups, two weeks after the first injection, treatment effect and satisfaction about the procedure was checked and second injection proceeded on condition. The satisfactory scores were measured on a 5 -grade scale $(0=$ no effect at all; $1=$ bad; $2=$ fair, 3 = good; 4 = excellent). "Excellent" meant "satisfied with the treatment result as expected". "Good" meant "not

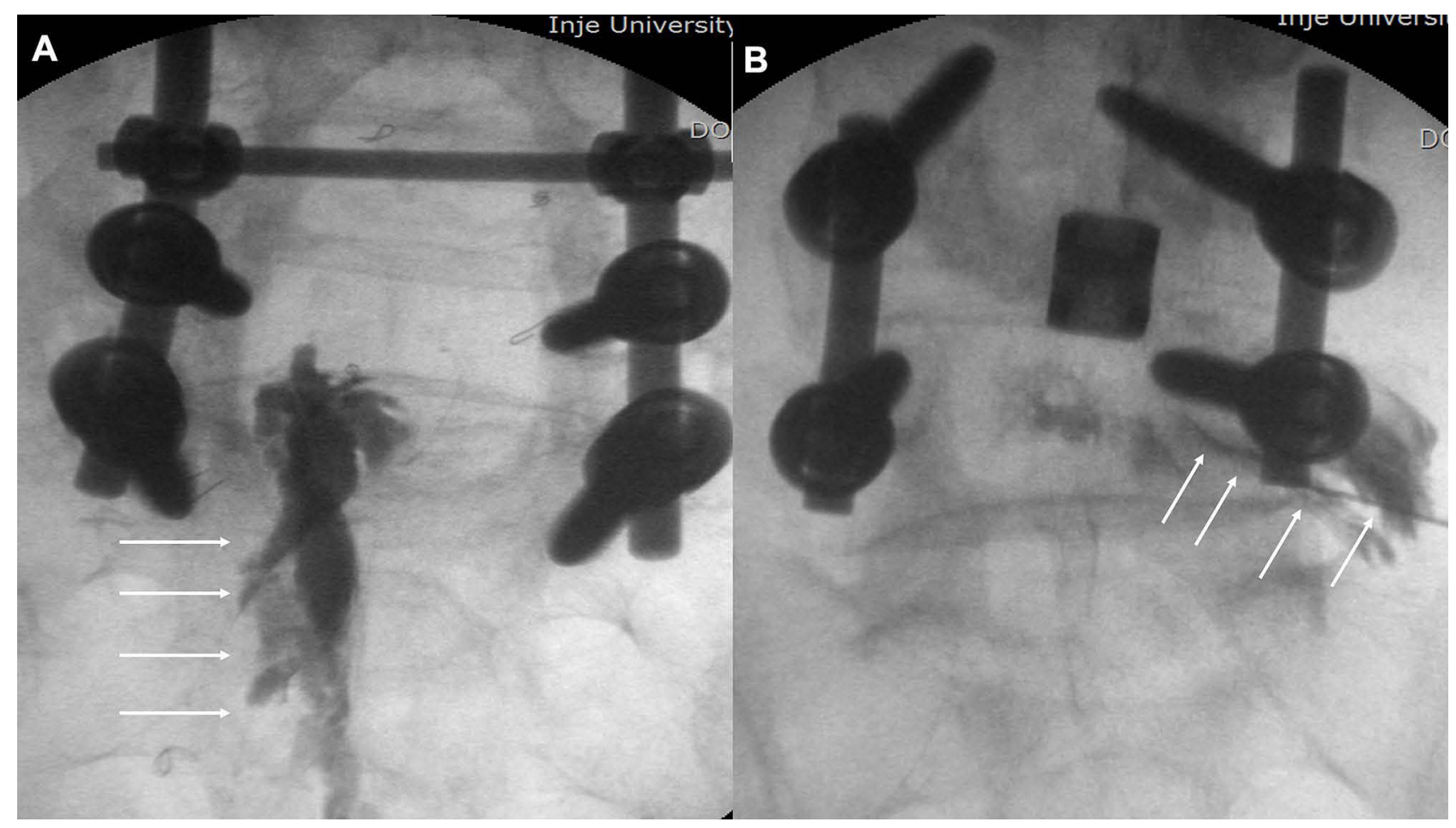

Figure I Fluoroscopy demonstrating different approaches of epidural steroid injection. (A) fluoroscopy-guided caudal epidural steroid injection. (B) fluoroscopy-guided transforaminal epidural steroid injection, The arrows indicates the epidural needle. 
as satisfied as expected but willing to try this treatment next time when pain redevelops". "Fair" meant "had some effect but not enough to choose the same treatment next time when pain re-develops". And finally, "Bad" meant "same effect as the prior treatment or worse." If the initial injection resulted in more than $50 \%$ reduction in verbal numeric pain scale score (VNS), the second injection was omitted and follow-up was scheduled. Also, if no pain relief or even pain deterioration was observed, a second injection was not considered. Only when the patients experienced pain relief but not more than $50 \%$ reduction in VNS and the patient satisfactory score was greater than 3; good at 2-week follow up, a second injection was scheduled. Since medications such as anti-inflammatory drugs and physical therapy did not have considerable effect on CPSS in the first place for 12 weeks, exercise programs or drug therapy besides intervention was not prohibited.

\section{Outcome Assessment}

Follow up parameters, the VNS and Oswestry Disability Index (ODI) were obtained by a nursing personnel not involved in the procedure at pretreatment, 1.3, and 6 months after the last injection. On the VNS, a score of 0 indicating no pain, and a score of 10 indicating the worst pain imaginable, in whole numbers with 11 integers. ${ }^{11}$ ODI is a commonly used disease-specific parameter for patients with LBP. ${ }^{12}$ ODI is calculated based on each score, which consists of ten items. Each of the items is scored from 0 to 5 , and the sum of each score is multiplied by 2 , thus, ODI ranging from 0 to 100 . An effective treatment was defined as a 2.5 points or more reduction in the VNS score, and 10 points or more reduction in ODI score at 1.3, and 6 months. ${ }^{13}$ Patients who did not meet the above conditions or who had to go through additional ESI or surgical treatment during follow up interval were considered as non-responders. In that case, VNS and ODI were confirmed for statistical analysis and excluded afterwards. Patients with a successful outcome were referred to as having received effective treatment, and was eligible for further follow up (Figure 2). Predictive variables were measured as follows: Patients' age was classified into five groups: <39-years-old, 40-49, 50-59, 60-69, and $>70$. The duration of radicular pain was also counted as a potential predictive variable which was classified as acute, $<6$ months or chronic $>6$ months. ${ }^{14}$ The medical chart was reviewed to evaluate occurrence of immediate adverse events such as vasovagal reaction or facial flushing. Also each patient had to fill out questionnaire at the end of procedure within 48 hours after each procedure and return it at the 2-week follow-up visit. The questionnaire evaluated the occurrence of severe complications such as occurrence of headaches, fever, transient pain exacerbation, hematoma, or aggravation of systemic metabolic diseases.

\section{Statistical Analysis}

The sample size was determined based on a review of previous studies and power analysis. Previous ESI studies set sample sizes of 20-30 participants per treatment group. On the other hand, this present retrospective study was designed as a non-inferiority trial. The mean VNS between two groups were presumed to be zero. A 1-point difference in the average with standard deviation of 2.0 of VNS between groups was set as the minimum needed for clinical relevance. With a power of $80 \%$, alpha of 0.05 , and drop out rate of $10 \%$, it was calculated that 55 patients were needed for each group to detect a 1-point difference in average VNS between two groups. The Chi-square test, Fisher's exact test, and Mann-Whitney U method were used to compare the basal demographic characteristics of the two groups. At each follow up, repeated measure analysis of variance (ANOVA) was used to compare VNS and ODI, and the Bonferroni correction was used for post-hoc comparison. The Chi-square test was used to evaluate the differences in proportions. Fisher's exact test was used when the expected value was less than 5. Chisquare test was performed to evaluate the relationship between possible outcome predictors and intervention effect. Logistic regression analysis was used to determine whether factors such as injection method, age, sex and the duration of pain were independent predictors of successful outcome. Statistical analyses were performed using SAS Enterprise Guide 4.1 (4.1.0.471) with significance level of $\mathrm{p}<0.05$.

\section{Results}

Of the total 94 FL-guided ESIs (CA $(\mathrm{n}=48)$ and TF $(\mathrm{n}=$ 46) performed during research period in our pain clinic, the inclusion criteria were met for 49 (52.1\%) cases. Twelve patients $(12.7 \%)$ who met the exclusion criteria were excluded. Thirty-three (35.1\%) patients were excluded because the patient did not return for follow-up. Finally, 21 patients in the CA group and 28 patients in the TF group were eligible for this study (Figure 2). The average age in each group was $60.5 \pm 10.4$ in the CA 


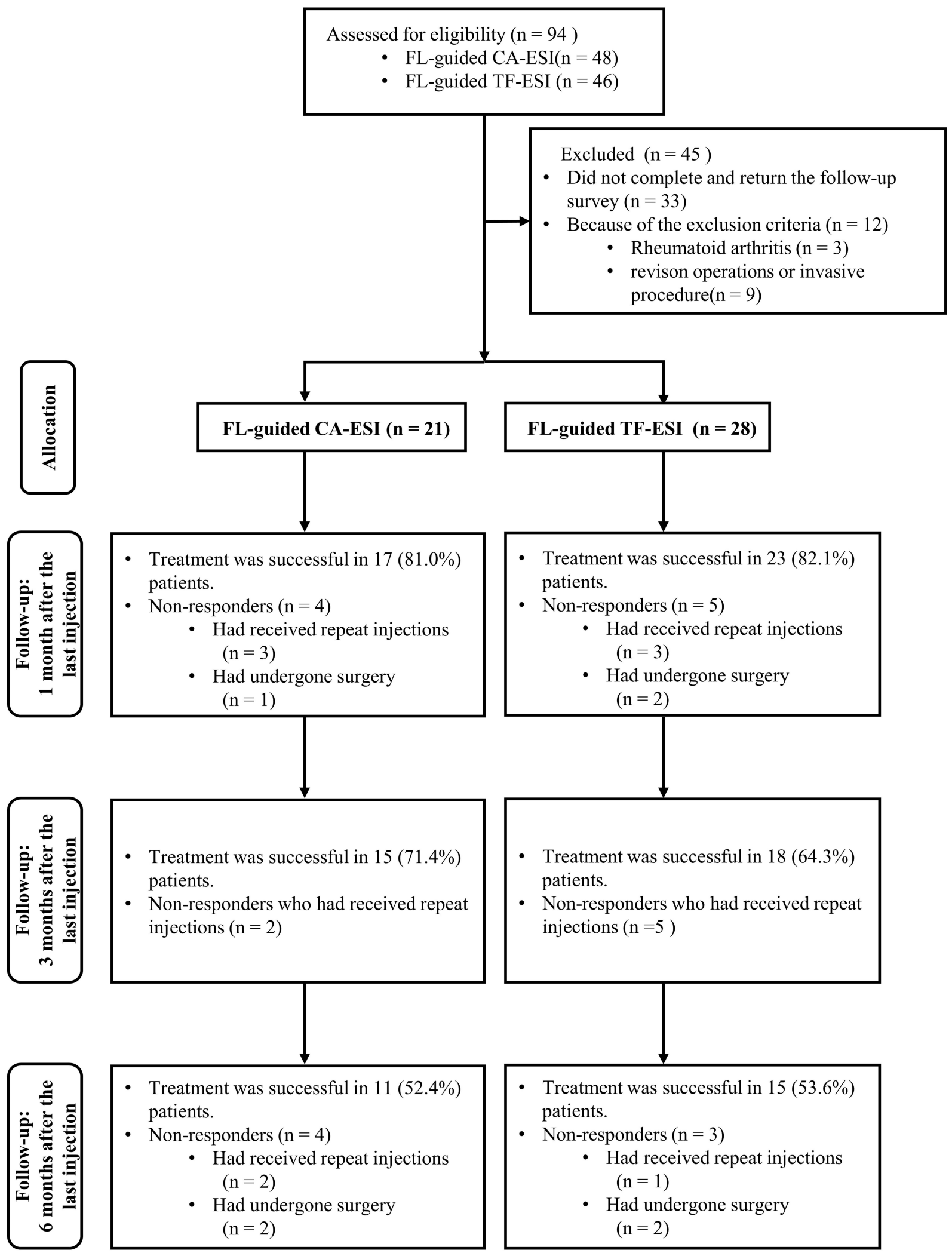

Figure 2 Flow diagram indicating progress of patients through the study.

Abbreviations: FL-guided CA-ESI, fluoroscopy-guided caudal epidural steroid injection; FL-guided TF-ESI, fluoroscopy-guided transforaminal epidural steroid injection. 
Table I General Characteristics of the Patients

\begin{tabular}{|c|c|c|c|}
\hline & $\begin{array}{l}\text { CA-ESI } \\
(n=2 I)\end{array}$ & $\begin{array}{l}\text { TF-ESI } \\
(n=28)\end{array}$ & P-value \\
\hline Age (years) & $60.5 \pm 10.4$ & $59.1 \pm 10.2$ & 0.644 \\
\hline \multicolumn{4}{|l|}{ Sex } \\
\hline Male & $6(28.6 \%)$ & 9 (32.1\%) & \\
\hline Female & 15 (7I.4\%) & 19 (67.9\%) & 0.843 \\
\hline BMI $\left(\mathrm{kg} / \mathrm{m}^{2}\right)$ & $25.99 \pm 3.04$ & $25.52 \pm 2.68$ & 0.211 \\
\hline $\begin{array}{l}\text { Pain } \\
\text { duration(Month) }\end{array}$ & $6.4 \pm 2.6$ & $6.8 \pm 2.2$ & 0.975 \\
\hline $\begin{array}{l}\text { Number of } \\
\text { injections }\end{array}$ & $\mid .48 \pm 0.5 \mathrm{I}$ & $1.46 \pm 0.50$ & 0.878 \\
\hline NSAID usage (\%) & $12(57.1)$ & $15(53.6)$ & \\
\hline Opioid usage (\%) & $7(33.3)$ & $10(35.7)$ & 0.804 \\
\hline \multicolumn{4}{|l|}{ Target root } \\
\hline L4 & $12(57.1 \%)$ & $17(60.7 \%)$ & \\
\hline L5 & 9 (42.9\%) & II (39.3\%) & 0.801 \\
\hline \multicolumn{4}{|l|}{ Posterior fixation } \\
\hline Fixation I level & II (52.4\%) & $15(53.6 \%)$ & \\
\hline Fixation 2 level & 7 (33.3\%) & II (39.3\%) & \\
\hline Fixation 3 level & $3(14.3 \%)$ & $2(7.1 \%)$ & 0.698 \\
\hline
\end{tabular}

Notes: Values are mean \pm standard deviation.

Abbreviations: BMI, body mass index; NSAID, non-steroidal anti-inflammatory drug.

group and $59.1 \pm 10.2$ in the TF group. No significant differences were observed in the general characteristics of age, sex, body mass index (BMI), number of injection, medication, pain duration and surgery (Table 1). VNS and ODI showed significant improvement at 1, 3, and 6 months in both groups. However, there was no significant differences of VNS and ODI score between groups at baseline and each follow up (Table 2). The proportion of patients with effective treatment $(2.5$ points or more improvement in the VNS score and 10 points or more improvement in the ODI) is illustrated in Figure 2, showing $52.4 \%$ in $\mathrm{CA}$ groups and $53.6 \%$ in $\mathrm{TF}$ groups at 6 months. There were no significant differences between the groups from 1 month follow up to 6 month follow up interval (Figure 3). The patient satisfactory scores, which were evaluated on the 2 week interval, demonstrated good and excellent rate being $85.7 \%(\mathrm{n}=18)$ among CA-ESI patients and $55.7 \%(\mathrm{n}=16)$ among TF-ESI patients $(\mathrm{p}<$ 0.05). In detail, 9 patients from the CA group gave score 4; "Excellent" and 3; "Good" respectively. One patient gave score 2; "Fair", and 2 patients gave score 1; "Bad" after the initial injection. In case of $\mathrm{TF}$ group $(\mathrm{N}=28), 6$ patients gave score 4; "Excellent" and 10 patients gave score 3; "Good". Six patients gave score 2; "Fair", 1 patient gave score 1; "Bad" and 1 patient gave score 0 ; "No effect at all". Injection method, sex, age, duration of pain and number of injection were not found to be statistically related to the effectiveness of FL-guided ESI. Univariate and multiple logistic regression analyses demonstrated that these factors were not independent predictors of effectiveness of FL-guided ESI ( $p>0.05)$. These results are summarized in Tables 3 and 4. There were no patients with the diameter of sacral hiatus less

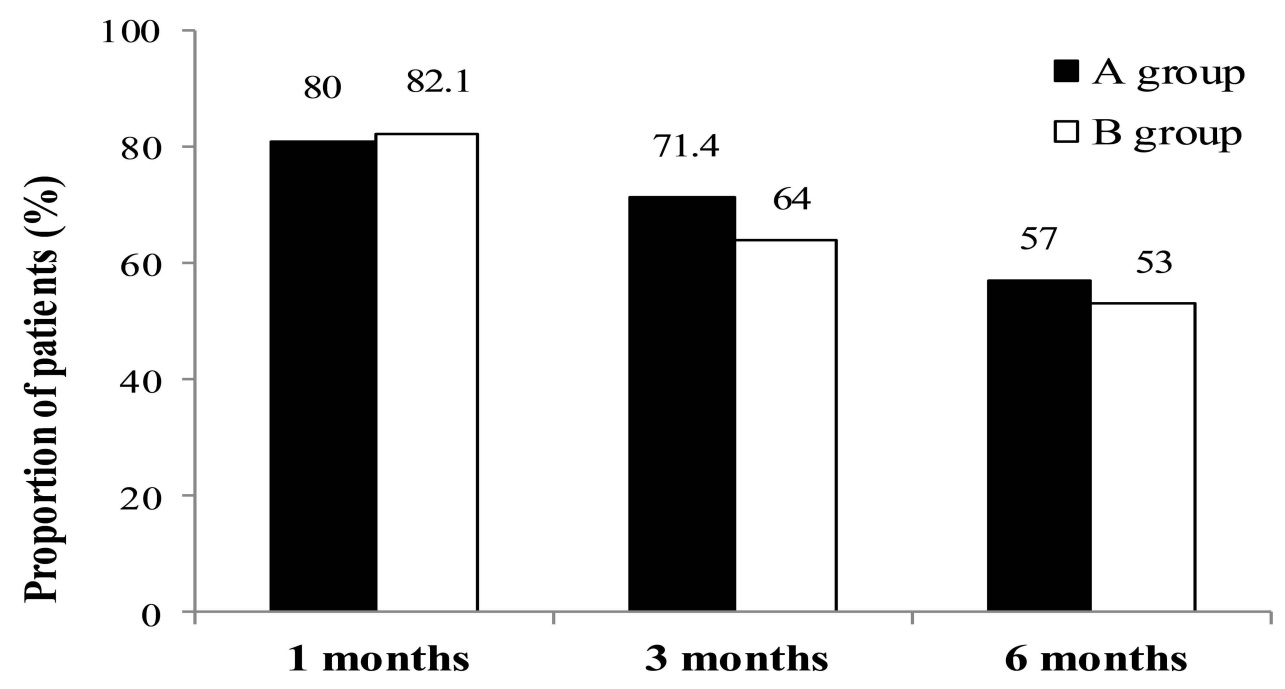

Figure 3 Illustration showing proportion of effective treatment in each group. Effective treatment was defined by significant pain relief ( $\geq 2.5$ points reduction in Verbal Numeric pain Scale from baseline) and functional improvement ( $\geq 10$ points reduction in Oswestry Disability Index from baseline). A group: Caudal epidural steroid injection, B group: Transforaminal epidural steroid injection. 
Table 2 Comparison of VNS and ODI from Baseline to I.3, and 6 Months After Last Injection

\begin{tabular}{|l|l|l|l|l|c|}
\hline & & Baseline & I Month & 3 Month & 6 Month \\
\hline VNS & CA & $6.43 \pm 0.74$ & $2.62 \pm 1.65^{*}$ & $2.85 \pm 1.26^{*}$ & $3.34 \pm 1.34^{*}$ \\
& TF & $6.48 \pm 0.80$ & $2.51 \pm 1.77^{*}$ & $2.99 \pm 1.45^{*}$ & $3.50 \pm 1.46^{*}$ \\
\hline \multirow{2}{*}{ ODI } & CA & $30.24 \pm 3.89$ & $15.33 \pm 6.95^{*}$ & $16.37 \pm 5.75^{*}$ & $17.78 \pm 4.97^{*}$ \\
& TF & $31.73 \pm 4.79$ & $16.52 \pm 8.23^{*}$ & $16.55 \pm 6.70^{*}$ & $18.22 \pm 6.17^{*}$ \\
\hline
\end{tabular}

Notes: ${ }^{*} p<0.05$ : Comparison before and after the injection. Values are mean \pm standard deviation.

Abbreviations: VNS, verbal numeric pain scale; CA, caudal approach; TF, transforaminal; ODI, Oswestry Disability Index.

Table 3 Univariate Analysis for Possible Outcome Predictors for Injection Effectiveness at Follow-Up

\begin{tabular}{|c|c|c|c|}
\hline Characteristic & $\begin{array}{c}\text { Effective } \\
(\mathrm{N}=26)\end{array}$ & $\begin{array}{l}\text { Ineffective } \\
\qquad(\mathrm{N}=23)\end{array}$ & $P$ value \\
\hline \multicolumn{4}{|c|}{ Injection method } \\
\hline CA (\%) & II (42.3) & $10(43.5)$ & \\
\hline TF (\%) & $15(57.7)$ & II (56.5) & 0.934 \\
\hline \multicolumn{4}{|l|}{ Gender } \\
\hline Male & 7 (26.9) & $8(34.8)$ & \\
\hline Female & $19(73.1)$ & $15(65.2)$ & 0.551 \\
\hline \multicolumn{4}{|l|}{ Age } \\
\hline$\leq 39$ & $0(0)$ & $\mathrm{I}(4.3)$ & \\
\hline $40-49$ & $4(15.4)$ & $3(13.0)$ & \\
\hline $50-59$ & $9(34.6)$ & $7(30.4)$ & \\
\hline $60-69$ & $8(30.8)$ & $8(34.8)$ & \\
\hline$>70$ & $5(19.2)$ & $4(17.4)$ & 0.804 \\
\hline \multicolumn{4}{|l|}{ Pain duration } \\
\hline$<6$ month & $12(46.2)$ & $7(30.4)$ & \\
\hline$>6$ month & $14(53.8)$ & $16(69.6)$ & 0.857 \\
\hline \multicolumn{4}{|c|}{ Number of injections } \\
\hline I & I4 (53.8\%) & $12(52.2 \%)$ & \\
\hline 2 & $12(46.9 \%)$ & II (47.8\%) & 0.907 \\
\hline
\end{tabular}

Abbreviations: CA, caudal approach; TF, transforaminal.

Table 4 Multiple Logistic Regression Analysis for Possible Outcome Predictors for Injection Effectiveness at Follow-Up

\begin{tabular}{|l|c|c|c|}
\hline Factor & OR & $\mathbf{9 5 \%} \mathbf{C I}$ & P value \\
\hline CA vs TF & 1.123 & $0.353-3.579$ & 0.844 \\
Sex & 0.999 & $0.944-1.057$ & 0.963 \\
Age & 0.701 & $0.200-2.458$ & 0.579 \\
Pain duration & 0.887 & $0.694-1.133$ & 0.336 \\
Number of injection & 1.036 & $0.323-3.322$ & 0.952 \\
\hline
\end{tabular}

Abbreviations: $\mathrm{OR}$, odds ratio; $\mathrm{Cl}$, confidence interval; $\mathrm{CA}$, caudal approach; $\mathrm{TF}$ transforaminal. than $2 \mathrm{~mm}$ or closed sacral hiatus. The amount of time used for the injection procedure was shorter in CA group than in TF group $(410.32 \pm 25.73$ seconds vs $640.65 \pm$ 18.03 seconds, $\mathrm{p}<0.05)$ Contrast medium pattern was confirmed in patients with possible data analysis. The highest contrast level on the epidurogram of CA group in lateral view was L5 in 8 patients (38.0\%), L4 in 9 patients (42.8\%), L3 in 2 patients $(9.5 \%)$ and $\mathrm{S} 1$ in 2 patients (9.5\%). Also, 4 patients of TF-ESI group, intravascular contrast spread was noticed without adverse symptoms. On the other hand, 4 patients in the CA group and 1 patient in the TF group had a vasovagal reaction immediately after injection. Four patients in the CA group and 3 patients in the TF group had a transient headache $(\mathrm{P}>0.05) .3$ in the CA group and 9 in the TF group reported transient pain exacerbation during the 2 week follow-up session. None of the patients had severe headache indicating post lumbar puncture syndrome, or other systemic complications such as decompensated heart disease or diabetes. Adverse events such as infection or hematoma were not recorded during the 2-week period after the procedure.

\section{Discussion}

The present study assessing treatment effects of FL-guided CA-ESI and TF-ESI showed clinically meaningful and statistically significant improvement in both pain and functional index at the end of mid-term follow up. Effective treatments (2.5 points or more improvement in the VNS score and 10 points or more improvement in the ODI) in $52.4 \%$ of CA-ESI group and $53.6 \%$ of TF-ESI were demonstrated at the end of the 6 month period. Proportion of successful treatments did not differ significantly between the groups at every follow up intervals. Consequently, pain reduction and functional improvement of FL-guided CA-ESI and TF-ESI were equally effective. When comparing 2 methods of TF and CA approaches, TF approach took longer FL time than in the CA group. This 
is likely due to the technical difficulty of TF approach compared to CA approach. Factors that generally complicates the spinal intervention are the presence of pathologies such as degenerative changes, anatomic variations, and they are more frequently encountered when using the TF approach. In addition, pedicle screw interferes the needle difficult to progress and lowers the accuracy of needle placement. ${ }^{15}$ If the FL time is prolonged, both the patient and the operator have a disadvantage of prolonged irradiation time. The TF approach also has a higher risk of complications compared to the CA approach. Spinal cord infarction is a rare but devastating complication of TFESI. Puncture of radiculomedullary artery or Adamkiewicz artery (AKA) during procedure has been implicated as the likely etiology. Thrombosis, embolism or from occlusion secondary to intravascular steroid suspension may lead to ischemia. ${ }^{16}$

The safe triangle, bordered by inferior margin of the pedicle, vertebral body, and upper portion of nerve root, was traditionally regarded safe during TF. ${ }^{10,17,18}$ However, in a retrospective study, AKA was identified in the safe triangle on digital subtraction spinal angiography. Hence, the AKA or the radiculomedullary vessels may be damaged by needle even in the safe triangle. In the case of CPSS patients, in addition to the AKA variant, there may be neovascularization at the injection site, and thus, steroid may be injected into the blood vessels during the intervention leading to vascular complications. ${ }^{19}$ Neovascularization resulting from scar tissue production after spine surgery around the spinal canal may originate from ramus of the anterior spinal artery. ${ }^{19}$ In the previous reports on paraplegia that occurred after ESI, a majority of cases were associated with prior history of lumbar spine surgery. ${ }^{19-21}$ Therefore, in the case of TF in CPSS patients, in addition to the presence of AKA, it is necessary to pay attention to the neovascularization and its atypical location during the procedure.

As for the satisfaction of treatment of patients, CA-ESI had higher satisfaction and less discomfort compared to TF-ESI after 2 weeks of evaluation. The pain or discomfort during procedure were also higher in TF-ESI groups than in CA-ESI group since the needle placed into the neural foramen was more likely to contact and irritate the nerve root or dorsal root ganglion in TF approach. ${ }^{22}$ Also, in CPSS patients various degenerative changes such as herniated disc, postoperative adhesions, thickened ligamentum flavum, spondylolisthesis and osteophyte formation may lead to intervertebral foramen stenosis. ${ }^{23}$ In the case of severe foraminal stenosis, discomfort or severe pain caused by the injected material can occur during the injection. Alongside more satisfaction and less discomfort during procedure, the CA-ESI has been reported to be a clinically effective management for CPSS. ${ }^{8}$ However, the CA-ESI has several drawbacks in comparison with the TF-ESI. First, the injectant may not reach the desired level in CA-ESI compared to TF-ESI, because the medication is administered via caudal hiatus, which is usually far from the spinal lesion in lumbar area. Therefore, to maximize the effect of intervention, a total volume of $20 \mathrm{~mL}$ mixture was administered. Previous studies reported that a volume of $20 \mathrm{~mL}$ reached $\mathrm{S} 1$ in $100 \%$ of patients, L5 in $89 \sim 91 \%$, L4 in $28.3 \% \sim 48 \%$ respectively. ${ }^{24,25}$

Secondly, ultrasound guidance was introduced in CA approach. However, if the diameter of sacral hiatus is $2 \mathrm{~mm}$ or less during the injection, needles larger than 22 $\mathrm{G}$ cannot be used. Also, CA-ESI cannot be done in case of anatomic deformity such as closed or small sacral hiatus. $^{25,26}$ Therefore, such a condition should be confirmed by ultrasound examination before injection treatment, and if necessary, injection should be performed using another injection method. In order to prepare for such a case, the state of anatomic variation should be confirmed by ultrasound before injection and other injection methods should be used if necessary. In the present study, there were no patients with the diameter of sacral hiatus less than $2 \mathrm{~mm}$ or closed sacral hiatus.

The current study had some limitations. The study was retrospective in design. Despite the fact that the subjects were selected according to the inclusion and exclusion criteria, there could have been heterogeneity among the subjects included in this study. Also, not enough patients estimated by power analysis were recruited for both groups, which requires further study with larger sample size. In addition, whether the patients received other treatment such as medication or physical therapy during follow-up periods was not considered. Since patients who were refractory to optional treatments were included and these treatments were assumed to have none or minimal effect on the outcome. Lastly, we compared only the procedure time and could not measure the degree of radiation exposure during the procedure. The longer the procedure, the greater the degree of exposure to radiation, but it is thought that accurate measurements will be needed in the future. Further prospective study with a larger sample size which supplements above limitations is necessary. 


\section{Conclusion}

In summary, the findings of present study suggest that the outcomes of FL-guided CA-ESI and TF-ESI for CPSS are equally effective in pain reduction and functional improvements. However, CA-ESI is associated with shorter procedure time which implies shorter radiation exposure time. In addition, compared with the TF-ESI, patients experience less discomfort during the injection, and the satisfaction with the procedure is confirmed to be better. Accordingly, both methods are effective, however, in the $\mathrm{CA}$ approach, patient satisfaction is higher and the procedure time is shorter.

\section{Abbreviations}

FL, fluoroscopy; TF, transforaminal; CA, caudal; ESI, epidural steroid injection; CPSS, chronic pain after spinal surgery.

\section{Acknowledgments}

The authors have not received financial support or any other benefits from commercial sources for the work reported in the manuscript.

\section{Disclosure}

The authors report no conflicts of interest in this work.

\section{References}

1. World Health Organization. ICD-11 for mortality and morbidity statistics (ICD-11 MMS)2018 version. World Health Organization. 2018 Available from https://icd.who.int/dev11/f/en\#http\%3a\%2f\%2fid.who. int $\% 2$ ficd\%2fentity\%2f345002890. Accessed July 5, 2021.

2. Slipman $\mathrm{CW}$, Shin $\mathrm{CH}$, Patel RK, et al. Etiologies of failed back surgery syndrome. Pain Med. 2002;3:200-214.

3. Hussain A, Erdek M. Interventional pain management for failed back surgery syndrome. Pain Pract. 2014;14:64-78.

4. Javid MJ, Hadar EJ. Long-term follow-up review of patients who underwent laminectomy for lumbar stenosis: a prospective study. J Neurosurg. 1998;89:1-7.

5. Chan C-W PP. Failed back surgery syndrome. Pain Med. 2011;12:577-606.

6. Manchikanti L, Singh V, Cash KA, Pampati V, Datta S. A comparative effectiveness evaluation of percutaneous adhesiolysis and epidural steroid injections in managing lumbar post surgery syndrome: a randomized, equivalence controlled trial. Pain Phys. 2009;12: E355-68.

7. Abdi S, Datta S, Trescot AM, et al. Epidural steroids in the management of chronic spinal pain: a systematic review. Pain Phys. 2007; 10:185-212.

8. Manchikanti L, Singh V, Cash KA, Pampati V, Datta S. Preliminary results of randomized, equivalence trial of fluoroscopic caudal epidural injections in managing chronic low back pain: part 3. Post surgery syndrome. Pain Phys. 2008;11:817-831.
9. Conn A, Buenaventura RM, Datta S, Abdi S, Diwan S. Systematic review of caudal epidural injections in the management of chronic low back pain. Pain Phys. 2009;12:109-135.

10. Park KD, Lee J, Jee H, Park Y. Kambin triangle versus the supraneural approach for the treatment of lumbar radicular pain. Am J Phys Med Rehabil. 2012;91(12):1039-1050. doi:10.1097/ PHM.0b013e318264573a

11. Hartrick CT, Kovan JP. Shapiro S The numeric rating scale for clinical pain measurement: a ratio measure? Pain Pract. 2003;3:310-316.

12. Fairbank JC. Pynsent PBThe Oswestry Disability Index. Spine (Phila Pa 1976). 2000;25:2940-2952.

13. Ostelo RW, de Vet HC. Clinically important outcomes in low back pain. Best Pract Res Clin Rheumatol. 2005;19(4):593-607.

14. Sontag MJ. A theoretical overview of the diagnosis and management of low back pain: acute vs chronic pain and themind/body continuum. In: Cole AJ, Herring SA, editors. The Low Back Pain Handbook: $A$ Guide for the Practicing Clinician. 2nd ed. Philadelphia: Hanley \&Belfus; 1993:39-48.

15. Kim SJ, Lee MH, Lee SW, Chung HW, Lee SH, Shin MJ. Radiation exposure for fluoroscopy-guided lumbosacral epidural steroid injections: comparison of the transforaminal and caudal approaches. J Spinal Disord Tech. 2014;27:E37-40.

16. Lyders EM, Morris PP. A case of spinal cord infarction following lumbar transforaminal epidural steroid injection: MR imaging and angiographic findings. Am J Neuroradiol. 2009;30:1691-1693.

17. Manchikanti L, Cash KA, Pampati V, et al. Evaluation of lumbar transforaminal epidural injections with needle placement and contrast flow patterns: a prospective, descriptive report. Pain Physician. 2004; 7:217-223

18. Murthy NS, Maus TP, Behrns CL. Intraforaminal location of the great anterior radiculomedullary artery (artery of Adamkiewicz): a retrospective review. Pain Med. 2010;11:1756-1764.

19. Wybier M. Transforaminal epidural corticosteroid injections and spinal cord infarction. Joint Bone Spine. 2008;75:523-525.

20. Houten JK, Errico TJ. Paraplegia after lumbosacral nerve root block: report of three cases. Spine J. 2002;2:70-75.

21. Huntoon MA, Martin DP. Paralysis after transforaminal epidural injection and previous spinal surgery. Reg Anesth Pain Med. 2004;29:494-495.

22. Lee JH, Shin KH, Bahk SJ, et al. Comparison of clinical efficacy of transforaminal and caudal epidural steroid injection in lumbar and lumbosacral disc herniation: a systematic review and meta-analysis. Spine J. 2018;18:2343-2353.

23. Baber Z, Erdek MA. Failed back surgery syndrome: current perspectives. J Pain Res. 2016;9:979-987.

24. Blanchais A, Le Goff B, Guillot P, Berthelot JM, Glemarec J, Maugars Y. Feasibility and safety of ultrasound-guided caudal epidural glucocorticoid injections. Joint Bone Spine. 2010;77:440-444.

25. Park Y, Lee JH, Park KD, Ahn JK, Park J, Jee H. Ultrasound-guided vs. fluoroscopy- guided caudal epidural steroid injection for the treatment of unilateral lower lumbar radicular pain: a prospective, randomized, single-blind clinical study. Am J Phys Med Rehabil. 2013;92:575-586.

26. Sekiguchi M, Yabuki S, Satoh K, Kikuchi S. An anatomic study of the sacral hiatus: a basis for successful caudal epidural block. Clin J Pain. 2004;20:51-54. 


\section{Publish your work in this journal}

The Journal of Pain Research is an international, peer reviewed, open access, online journal that welcomes laboratory and clinical findings in the fields of pain research and the prevention and management of pain. Original research, reviews, symposium reports, hypothesis formation and commentaries are all considered for publication. The manuscript management system is completely online and includes a very quick and fair peer-review system, which is all easy to use. Visit http:// www.dovepress.com/testimonials.php to read real quotes from published authors. 